\title{
Electrophysical properties of meso-porous silicon free standing films modified with palladium
}

\author{
A.I. Manilov, V.A. Skryshevsky, S.A. Alekseev, G.V. Kuznetsov \\ Institute of High Technologies \\ Taras Shevchenko Kyiv National University, \\ 64, Volodymyrska str., 01601 Kyiv, Ukraine, \\ E-mail:an.manilov@yandex.ru
}

\begin{abstract}
Resistivity and complex impedance voltage dependences for thick mesoporous silicon free layers were studied in this work. The asymmetrical by the sign of applied voltage experimental curves at low frequencies have been obtained. Modification of electrophysical properties due to introduction of palladium particles into the porous matrix is observed. Impedance change regularities during oxidation of the samples have been measured. The explanation of experimental results by asymmetrical distribution of charge carrier traps in the bulk of porous silicon has been suggested. Energy band diagrams and charge transfer mechanisms of these heterostructures are discussed.
\end{abstract}

Keywords: meso-porous silicon, palladium particles, impedance.

Manuscript received 17.11.10; accepted for publication 02.12.10; published online 28.02.11.

\section{Introduction}

It is well know that electrophysical and optical properties of porous silicon (PS) essentially depend on its chemical composition and morphology [1,2]. Main impurities of as-prepared PS are hydrogen and fluorine that passivate dangling silicon bonds. During aging of the samples in air, fluorine is lost; quantity of adsorbed oxygen is essentially increased (that causes oxidation of material), as well as $\mathrm{CO}_{\mathrm{x}}$ groups $[1,3]$.

Oxidation of PS during natural or artificial aging is accompanied with essential modification of its electrophysical properties. In the course of it, either increasing or decreasing conductivity of the samples is registered, depending on their species and experimental conditions $[4,5]$. Differences in results of recent several publications indicate necessity of further researches in this field.

Structures, based on PS and Pd, attract attention due to possible applications as sensors of hydrogencontaining gases [6-8], solid-state materials for hydrogen storage $[9,10]$. Electrophysical and sensor properties of these heterostructures are under influence of the used particles or films of $\mathrm{Pd}$, various amounts of metal $[11,12]$. Similar regularities are present in hydrogen sources based on PS and Pd where the difference in hydrogen capacity and extraction was registered [13]. So, analysis of the influence of Pd particles deposited in different quantities on electrophysical properties of PS is a quite important task.

Also, in publications the heterostructures metal/PS layer/c - Si /metal are usually investigated [14]. Less attention was paid for free standing films of PS, which could be effectively used in hydrogen energy $[10,13]$, sensory and micro-engineering $[15,16]$.

\section{Experimental}

PS was produced by electrochemical etching $\left(100 \mathrm{~mA} / \mathrm{cm}^{2}\right)$ of silicon wafers $\left(\mathrm{p}^{++},(100)\right.$ orientation, $\Omega=0.001 \mathrm{Ohm} \cdot \mathrm{cm})$ in a $48 \% \mathrm{HF}$ :ethanol solution (volume ratio 1:1) for 2 hours. At the end of anodization, the PS film was separated from the substrate using a high current pulse $\left(1 \mathrm{~A} / \mathrm{cm}^{2}, 5 \mathrm{~s}\right)$. In this way, freestanding meso-PS layers were created with $55 \%$ porosity and a thickness of approximately $300 \mu \mathrm{m}$. A part of the PS sample was no longer processed and used as the reference one.

To incorporate the palladium into PS matrix, we used chemical deposition technology [13]. The PS free layers were washed in a $\mathrm{HF}(48 \%)$ :ethanol solution (1:9) for 5 minutes to remove the surface oxide, washed in ethanol and then immersed for 1 hour in an acetonitrile solution of palladium acetate $\left(\mathrm{Pd}\left(\mathrm{CH}_{3} \mathrm{CO}_{2}\right)_{2}\right) .1 \mathrm{ml}$ of the $\mathrm{Pd}\left(\mathrm{CH}_{3} \mathrm{CO}_{2}\right)_{2}$ solution was used for $10 \mathrm{mg}$ of PS, and the concentrations used were 0.001 and $0.01 \mathrm{mmol} / 1$ for 
preparation of $\mathrm{PS}+1 \% \mathrm{Pd}$ and $\mathrm{PS}+10 \% \mathrm{Pd}$ samples, respectively. After $\mathrm{Pd}$ deposition, the samples were washed in acetonitrile and then dried in ambient air.

Induced aging (oxidation) was made using thermal treatment of PS samples in ambient atmosphere for 30 minutes. Annealing temperatures were 200, 350, 450 and $600{ }^{\circ} \mathrm{C}$. The samples of porous $\mathrm{SiO}_{2}$ were produced by full oxidation of PS free layers in $\mathrm{H}_{2} \mathrm{O}$ :EtOH: $\mathrm{NH}_{3}(10 \%)$ solution, afterwards dried in air for a long period of time.

Surface of the samples is rough from the top side of the free layer and smooth from the back side (due to separation of PS film from silicon slab). Electrical contacts were made using thermal deposition of Ni film. Short description of studied samples is presented in Table 1.

Current-voltage $(I-V)$ curves were measured by potentiostat PI - 50 connected with AD-DA PC plate ET1250 to personal computer. Complex impedance measurements were performed using the device E $7-20$ within the frequency range $50 \mathrm{~Hz}$ to $1 \mathrm{MHz}$, with signal magnitude $0.1 \mathrm{~V}$. The back side of the samples (smooth surface) was under zeroth potential.

\section{Results and discussion}

\subsection{Impedance-voltage characteristics of PS free layers}

PS free layers have high electrical resistivity. That is explained mainly by technological depletion of PS, due to trapping the electrons on surface states and passivation of dopants with hydrogen atoms $[1,2]$. Also, interfaces between grains lead to creation of potential barriers on the grain boundaries, which complicate charge transport through the structure [17]. Furthermore, metal contacts cause additional potential barriers on the interfaces with PS [14]. So, it is quite difficult to create an appropriate equivalent circuit. Usual measurements of capacitance and resistivity, based on parallel or sequential circuit, are inapplicable in this case. Therefore, complex impedance measurements were provided.

Resistivity of the PS samples measured in DC mode has values up to several MOhm. Positive and negative branches of the $I-V$ characteristics are similar, but existing asymmetry is expressed in differences between current values inherent to positive and negative voltages. However, the obtained values of direct current were close to the sensitivity limit of the used experimental devices.

Table 1. Types of the samples.

\begin{tabular}{|c|c|c|}
\hline & Rough side (top) & Smooth side (back) \\
\hline PS, oxidized & & $\begin{array}{c}\text { Ni complete } \\
\text { metallization }\end{array}$ \\
\cline { 1 - 1 } PS $\mathrm{SiO}_{2}$ & & Ni contacts \\
& (diameter $1 \mathrm{~mm}$ ) & $\begin{array}{c}\text { Pd island-type } \\
\text { metallization }\end{array}$ \\
\cline { 1 - 1 } & & $\begin{array}{c}\text { Pd complete } \\
\text { metallization }\end{array}$ \\
\hline
\end{tabular}
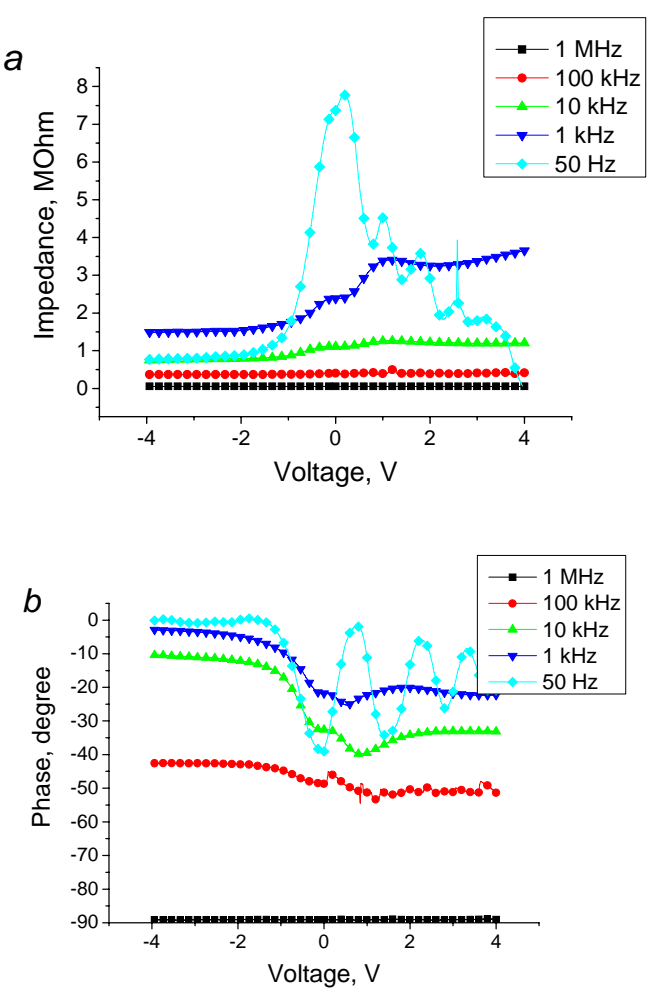

Fig. 1. Voltage dependences of the impedance (a) and phase (b) for the initial samples of PS free layers and various signal frequencies.

Voltage dependences of the impedance value inherent to these PS free layers for different signal frequencies are presented in Fig. 1a. It is obtained that experimental curves at the high frequencies $(1 \mathrm{MHz})$ are symmetrical, and impedance is almost independent from voltage. At the same time, at lower frequencies $(1-10 \mathrm{kHz})$ the impedance value for a positive bias increases, and a maximum appears on the curve. So, in symmetrical, at first sight, samples the asymmetrical voltage dependences occur.

The phase-voltage characteristics of the PS free layers are displayed in Fig. 1b. At $1 \mathrm{MHz}$, the phase value is constant and close to $-90^{\circ}$, i.e. the sample behavior looks like that of a uniform capacitor. So, charge transfer through the bulk is negligibly small. Decrease in the signal frequency leads to decrease in the phase absolute value, and an asymmetrical form of the curve is developed. So, electron transfer through the bulk is emerged, which causes energy dissipation. Evidently, it is indicative of the fact that electron states play an important role in charge transport mechanisms and electrophysical properties of the samples.

\subsection{Energy band scheme and transport mechanisms of PS free layers}

Explanation of charge transport mechanisms and asymmetry of the impedance-voltage curve could be given with the energy band scheme illustrated in Fig. 2. 
Quantum-size effects in meso-PS are not observed, so the band gap width is constant $[14,17]$. Though, the dopants have not been moved off during anodization of degenerated silicon (concentration of shallow energy levels is kept close to $10^{19} \mathrm{~cm}^{-3}$ ), PS gets high resistance due to effect of compensation. Compensation of obtained semiconductor and shift of the Fermi level to the middle of the band gap are explained by trapping the electrons at the surface states, which concentration is $10^{12}-10^{14} \mathrm{~cm}^{-2} \mathrm{eV}^{-1} \quad$ [17]. Coulomb interaction between positive charged donors and negative charged surface states leads to modulation of the bands and appearance of the so-called "states' concentration tails" near band boundaries. Due to a large thickness of the high resistive samples $(300 \mu \mathrm{m})$, Schottky barriers on the interfaces contact-PS have almost no influence on the electrophysical properties of the structure.

From the results of P. 3.1, it is clear that transfer of charge carriers through the bulk of PS layers exists only at relatively low frequencies. It determines that the main charge transport mechanism in this case is hopping through states in the band gap. Additionally, the current of free carriers over the percolation level in conduction band is possible $[14,17]$.

The difference in the impedance and resistance values for positive and negative voltages could be explained by the following reasons. There are different treatments of front and back sides of the free standing film (see P. 2.1, Table 1). So, it causes an asymmetrical distribution of charge carrier traps ( $\left.N_{\text {traps }}\right)$ in bulk of the samples. It is possible to suppose that near the top side of the sample with rough surface the density of energy states in the band gap is higher than that near the bottom side (smooth surface). Charge distribution over the sample is under influence of distribution of electrons on the traps $\left(Q_{\text {traps }}\right)$. In the equilibrium state, distribution of electrons over the traps is uniform (Fig. 2a). Due to asymmetrical distribution of traps, an essential quantity of free energy states appears in the top part of the sample. After applying the external voltage, transfer of charge carriers begins: through traps near the Fermi level (hopping mechanism), over percolation level in conduction band, and filling the free traps in the top part of the sample with electrons from the valence band (Fig. 2b). Situations for positive and negative biases are similar. Due to above mentioned processes, asymmetrical distribution of electrons on the traps occurs. Then, the quantity of electrons and corresponding negative charge density on the traps decrease from top to bottom of PS free layer. So, the internal negative difference of potentials $\left(V_{\text {internal }}\right)$ exists (Fig. 2c). This effect leads to amplification of the external voltage for negative biases and to attenuation for the positive ones. Thereof, the difference in impedance values appears from a lower magnitude for negative biases to a higher magnitude for the positive ones. The maximum on impedance curves could be associated with recharging processes in charge carrier traps.
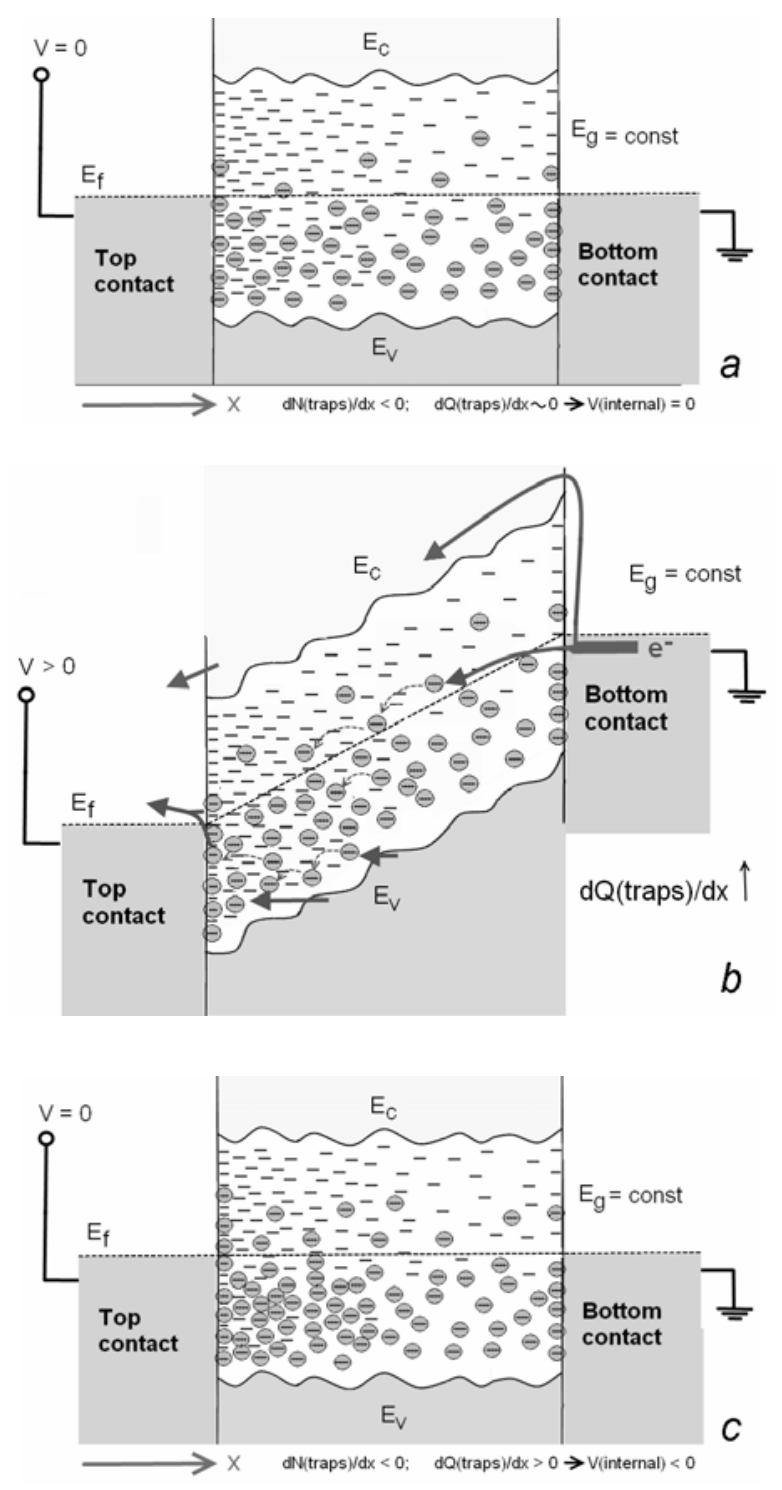

Fig. 2. Energy band scheme and charge transfer mechanisms of the structures metal/thick PS layer/metal. (a) - equilibrium state without external voltage; (b) - charge transfer processes at a positive bias; (c) - non-equilibrium state after dispose of the external voltage.

Also, the asymmetry of impedance could be presumably explained by different areas of top and bottom contacts of the samples, or asymmetry in modulation of bands. However, first of these ways influence of the contacts - should be minor due to the essential thickness and high resistance of PS layer, so planar currents are negligibly small. Another way asymmetry in modulation of bands - could cause modification of free carrier transport over the percolation level in conduction band regularities. But, this effect should be under influence of recharging the electron traps, so it is associated with the main explanation by asymmetrical distribution of charge carrier traps in the bulk of PS. 


\subsection{Influence of Pd introduction on electrophysical properties of PS free layers}

High electrical resistance of the PS free layers is essentially reduced after introduction of Pd particles, as intense as higher is the quantity of metal. In this case, $I-V$ characteristics of $\mathrm{PS}+1 \% \mathrm{Pd}$ become more symmetrical as compared to those of pure PS, whereas for PS $+10 \%$ Pd asymmetry increases.

Voltage dependences of the impedance value and phase for the samples for different signal frequencies are adduced in Fig. 3. It could be seen that in the samples of $\mathrm{PS}+1 \% \mathrm{Pd}$ the difference of the impedance value between negative and positive polarities is almost absent as compared to that of pure PS. In this case, the samples of PS $+10 \%$ Pd display an increase in this effect. These are in accord with regularities of the $I-V$ characteristics. As it follows from the phase figures (Fig. 3b,d) this increase in the Pd content causes growth of the active component of resistivity, which becomes
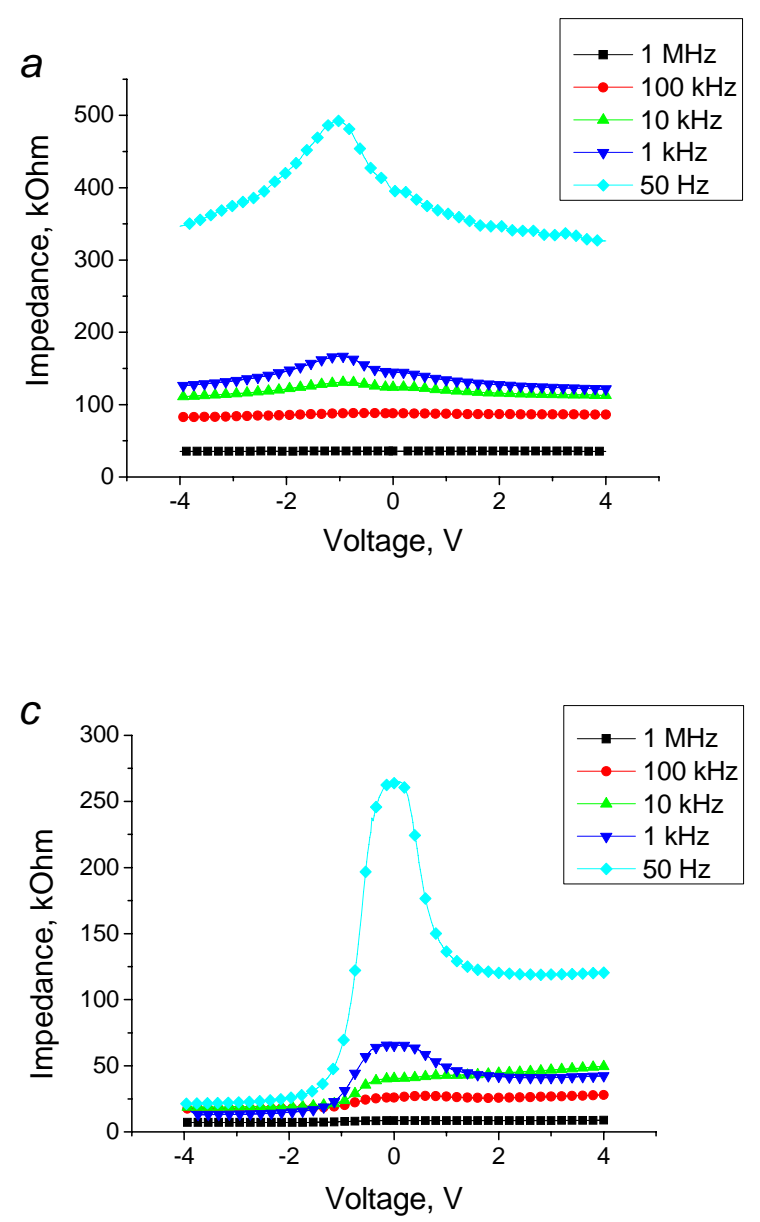

apparent already at $1 \mathrm{MHz}$. So, charge transfer through the samples increases.

It could be explained by the following reasons. Firstly, introduction of $\mathrm{Pd}$ particles into the porous matrix leads to decrease in the effective width of PS layer, in this case, the top and bottom layers of Pd are not overlapped (which was checked with optical microphotographs of cleaved facets in the samples). Secondly, $\mathrm{Pd}$ deposition on $\mathrm{Si}$ causes existence of additional defect states that penetrate into bulk of PS up to the depth $0.5 \mu \mathrm{m}$ [18]. Accordingly, distribution of the surface states density is modified (see Fig. 2) and quantity of energy states in the band gap is increased, which leads to easing the hopping transport. It is possible that island-type metallization of the bottom side of PS $+1 \%$ Pd leads to balancing the concentration of electron states near the top and bottom of the sample, which causes the symmetrical impedance dependence on voltage. At the same time, complete metallization of PS $+10 \%$ Pd sample bottom and essential penetration of
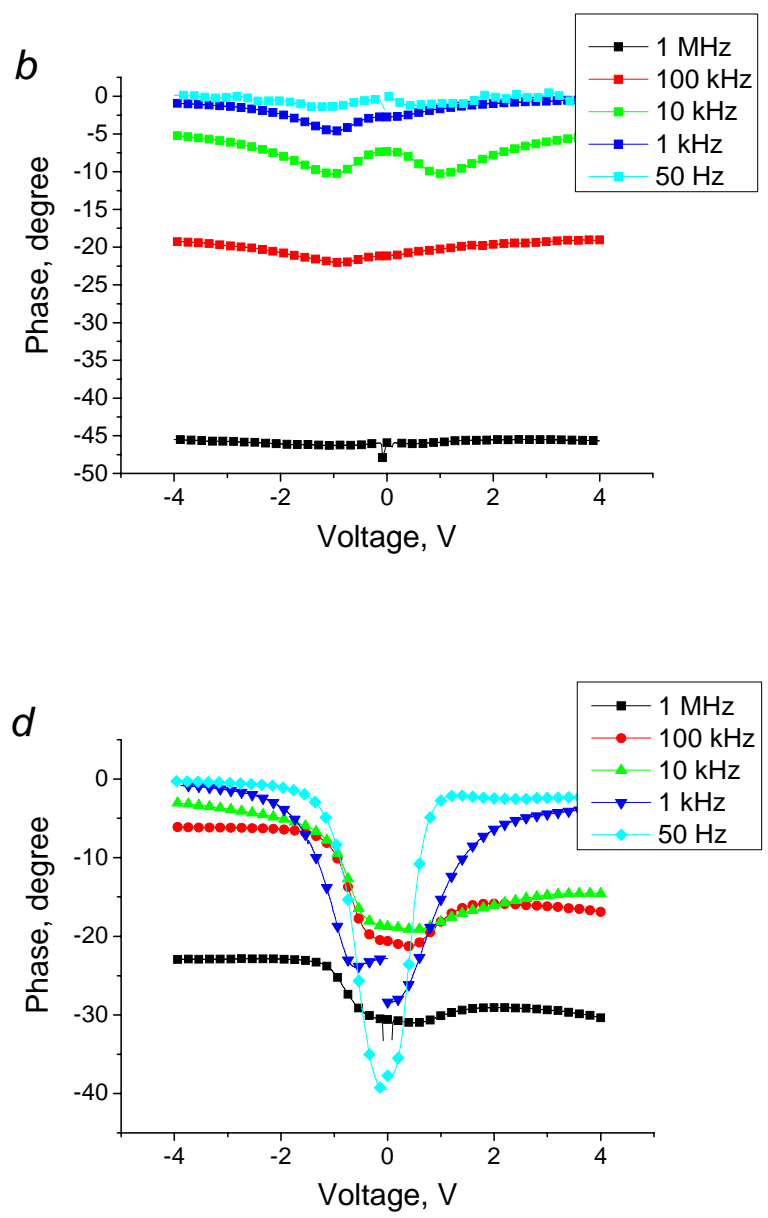

Fig. 3. Voltage dependences of the impedance and phase at various signal frequencies for the samples of PS $+1 \% \mathrm{Pd}(\mathrm{a}-\mathrm{b})$, PS $+10 \%$ Pd $(\mathrm{c}-\mathrm{d})$. 
Pd particles deeply into the porous matrix from both sides does not change the effect observed in pure PS, but reinforce it due to a less effective width of the sample. Thirdly, introduction of the metal particles leads to a greater role of barrier effects on contacts and emissive component of charge transport. It affects the presence of active resistance component at high frequencies.

\subsection{Influence of annealing on electrophysical properties of PS free layers}

Annealing of PS causes an essential increase in resistivity of the samples (Fig. 4). Moreover, the impedance value changes more than 1-2 orders already after heating at $200{ }^{\circ} \mathrm{C}$. Note that in several publications $[4,5]$ an opposite effect was observed - a decrease in resistance, which was explained by destruction of $\mathrm{Si}-\mathrm{H}-\mathrm{B}$ complexes at $150{ }^{\circ} \mathrm{C}$. Results of our experiment could be explained by essential oxidation of the samples that converts them into the dielectric state and substantially reduce the conductivity of this structure. It is confirmed with $Z(V)$ measurements (for $1 \mathrm{kHz}$ and $1 \mathrm{MHz}$ frequencies), when $Z$ becomes almost independent from voltage after annealing of the samples.
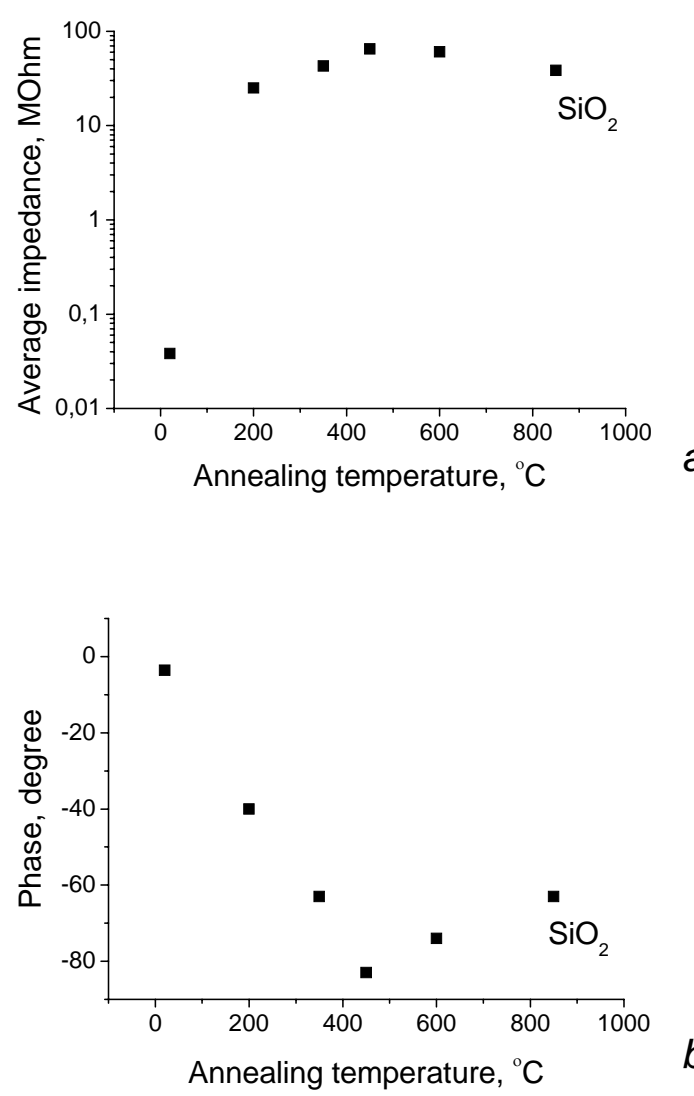

Fig. 4. Average values of the impedance (a) and phase (b) on $1 \mathrm{kHz}$ for PS free layers for various annealing temperatures and $\mathrm{SiO}_{2}$. Regularities for $1 \mathrm{MHz}$ are the same.
At annealing temperatures over $450{ }^{\circ} \mathrm{C}$, the decrease in resistance of the structures is observed (comparing to that of lower heating temperatures). Especially, it is noticeable for pure $\mathrm{SiO}_{2}$. This effect could be explained by densification and greater stoichiometry of silicon oxide after essential oxidation. So, conductivity increases as compared to that of friable state at lower annealing temperatures.

\section{Conclusions}

Impedance and resistivity of meso-porous silicon free layers have asymmetrical voltage dependences at low frequencies. It could be explained by asymmetrical distribution of charge carrier traps in bulk of the samples and a leading role of the hopping charge transport mechanism.

Annealing of PS free layers leads to increasing the impedance up to 1-2 orders of magnitude. It could be explained by deeper oxidation of the samples, which transforms PS into the dielectric state.

Introduction of Pd particles into the PS free layers resulted in growth of conductivity, decrease (for $\mathrm{PS}+1 \% \mathrm{Pd}$ ) or increase (for $\mathrm{PS}+10 \% \mathrm{Pd}$ ) of the difference between impedance values for positive and negative voltage biases. It could be explained with the following consequences of penetration of $\mathrm{Pd}$ particles into the porous matrix: (a) decrease in the PS layer effective thickness, (b) creation of new electron traps and corresponding change in distribution of energy states within the band gap of PS, (c) an increasing role of barrier effects on Pd - PS contacts.

\section{References}

1. V. Parkhutik, Porous silicon - mechanisms of growth and applications // Solid-State Electronics, 43, p. 1121-1141 (1999).

2. O. Bisi, S. Ossicini, L. Pavesi, Porous silicon: a quantum sponge structure for silicon based optoelectronics // Surf. Sci. Repts., 38, p. 1-126 (2000).

3. A.M. Orlov, A.A. Skvortsov, A.G. Klementyev, A.V. Sindyaev, Adsorptive modifications on porous silicon surface during natural and high temperature aging // Pis'ma zhurnal tekhnich. fiziki, 27(2), p. 76-83 (2001), in Russian.

4. S.P. Zimin, A.N. Bragin, Conductivity relaxation in coated porous silicon after annealing // Semiconductors 33 (4), p. 457-460 (1999).

5. D.I. Bilenko, O.Ya. Belobrovaya, E.A. Jarkova, I.B. Mysenko, E.I. Khasina, The influence of the adsorption on the electrophysical properties of the structures on the base of oxidized porous silicon // Fizika tekhnika poluprovodnikov, 36(4), p. 490-495 (2002), in Russian.

6. V.A. Skryshevsky, V. Polischuk, A.I. Manilov, I.V. Gavrilchenko, R.V. Skryshevsky, Improved 
hydrogen detection of island type palladium film nanoporous silicon diode at room temperature // Sensor Electronics and Microsystem Technologies, 2, p. 21-27 (2008).

7. T.I. Gorbanyuk, A.A. Evtukh, V.G. Litovchenko, V.S. Solntsev, E.M. Pakhlov, Adsorption of hydrogen containing molecules in multilayer structures with films of porous silicon and palladium // Physics and Chemistry of Solid State 7 (1), p. 60-66 (2006).

8. S. Bravina, N. Morozovsky, R. Boukroub, Dynamic electrophysical characterization of porous silicon humidity sensing // Semiconductor Physics, Quantum Electronics \& Optoelectronics, 9 (1), p. 79-83 (2006).

9. V. Lysenko, F. Bidault, S. Alekseev, V. Zaitsev, D. Barbier, Ch. Turpin, F. Geobaldo, P. Rivolo, E. Garrone, Study of porous silicon nanostructures as hydrogen reservoirs // J. Phys. Chem. B, 109, p. 19711-19718 (2005).

10. A.I. Manilov, S.V. Litvinenko, S.A. Alekseev, G.V. Kuznetsov, V.A. Skryshevsky, Use of powders and composites based on porous and crystalline silicon in the hydrogen power industry // Ukrainian Journal of Physics, 55 (8), p. 929-936 (2010).

11. K. Luongo, A. Sine, S. Bhansali, Development of a highly sensitive porous Si-based hydrogen sensor using Pd nanostructures // Sensors and Actuators B, 111-112, p. 125-129 (2005).
12. V.G. Litovchenko, T.I. Gorbanyuk, O.O. Yefremov, A.A. Yevtukh, Yu.G. Ptushinskyy, V.A. Ischuk, O.V. Kanash, Catalytic peculiarities of ultra-thin palladium films and its alloys // Ukrainian Journal of Physics 48 (6), p. 565-575 (2003).

13. A.I. Manilov, S.A. Alekseev, V.A. Skryshevsky, S.V. Litvinenko, G.V. Kuznetsov, V. Lysenko, Influence of palladium particles impregnation on hydrogen behavior in meso-porous silicon // $\mathrm{J}$. Alloys and Compounds 492, p. 466-472 (2010).

14. S.P. Zimin, Classification of porous silicon electrical properties // Fizika tekhnika poluprovodnikov, 34(3), p. 359-363 (2000), in Russian.

15. I. Bazrafkan, R.S. Dariani, Electrical behavior of free-standing porous silicon layers // Physica B: Condensed Matter, 404 (12-13), p. 1638-1642 (2009).

16. A.Yu. Karlash, A.I. Manilov, I.I. Ivanov, V.A. Skryshevsky, Diffusion-adsorption dynamics in multilayer silicon quantum-dimension structures // Abstracts of III Ukrainian Conference on Semiconductor Physics, Odessa, 2007, p. 244.

17. V.A. Skryshevsky, Physical Basics of Semiconductor Chemical Sensors. Kyiv, Kyiv University Press, 2006.

18. I.A. Karpovich, S.V. Tikhov, E.L. Shobolov, I.A. Andryuschenko, Defect formation in GaAs and $\mathrm{Si}$ at $\mathrm{Pd}$ deposition on the surface // Fizika tekhnika poluprovodnikov, 40 (3), p. 319-323 (2006), in Russian. 